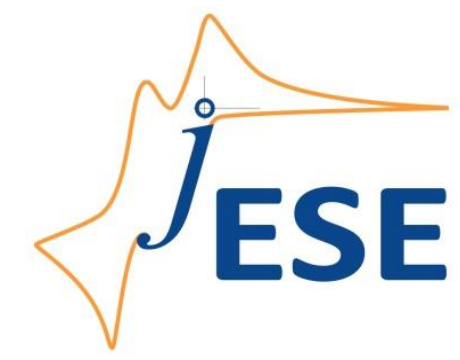

Open Access : : ISSN 1847-9286

www.jESE-online.org

Original scientific paper

\title{
Electrochemical surface modification technique to impede mild steel corrosion using perfluorooctanoic acid
}

\author{
Shubha H. Nataraj, Venkatesha T. Venkatarangaiah ${ }^{凶}$, Anantha N. Subba Rao \\ Department of Studies in Chemistry, School of Chemical Sciences, Kuvempu University, \\ Shankaraghatta-577451, Karnataka, India \\ ${ }^{\square}$ Corresponding Author: drtvvenkatesha@yahoo.co.uk, Tel:+91-9448855079; Fax: +91-08282-256255
}

Received: August 17, 2015; Revised: January 8, 2016; Accepted: February 8, 2016

\begin{abstract}
The present work demonstrated that corrosion inhibition efficiency of electrochemically generated organic coat is remarkably more effective than self-assembled monolayer (SAM) generated by dip coating technique. Perfluorooctanoic acid (PFOA) is used to modify mild steel surface for effective protection. Infrared reflection absorption spectroscopy and contact angle measurements substantiate the modification of mild steel surface and its effect on surface hydrophobicity. A comparison between electrochemical properties of PFOA SAM generated by dip coat method (DC-PFOA) and PFOA coat generated by electrochemical method (EC-PFOA) is presented. Electrochemical measurements reveal that the corrosion protection efficiency of $E C$ PFOA (91\%) is much superior to DC-PFOA (28\%).
\end{abstract}

\section{Keywords}

Self-assembled monolayer; Mild steel; Contact angle; IRRAS; Corrosion; Electrochemical coating.

\section{Introduction}

Mild steel is most frequently used engineering metallic material owing to its excellent mechanical - material property, availability and relative low cost [1]. The corrosion of mild steel is a universal problem and poses major economical and safety threats. Protective coatings [2] and inhibitors [3,4] are the most commonly used method of corrosion control. Blocking anodic and cathodic sites on the surface is a smart approach to impede the corrosion process. This can be achieved by modifying the metal surface at molecular level using organic molecules to produce a protective layer to avoid the invasion of corrosive agents [5-7].

The self-assembled monolayer (SAM) technique has gained a lot of attention in this regard, because it imparts hydrophobicity to the surface. The SAM of organic molecule is generated by the 
immersion of substrate in a solution containing adsorbate molecule (dip coating). Here, the molecular assembly takes place without the application of any external force i.e., pressure [8]. To generate SAM, organic molecule should contain one hydrophilic head group and hydrophobic tail group, where the hydrophilic group attach to the surface and hydrophobic group extend towards the space. Thus it forms barrier film over substrate and controls the diffusion of corrosive agents [9]. K. Nozawa et. al. modified iron surfaces by alkanethiole monolayer and studied its corrosion property [10]. Paul E. Hintze et. al. studied corrosion behavior of modified aluminium surface by organosilane SAM [11]. In an earlier paper we reported that sodium oleate SAM protects mild steel from corrosion in saline water [12].

PFOA is a synthetic anionic surfactant with hydrophilic acid head group and hydrophobic as well as lipophobic fluorinated carbon chain. PFOA has excellent chemical stability, thermal stability, strong water repulsive and oil repulsive properties. Hence, It is used in the leather stuffs as water proof repellents, as insulators in electric wires, in hydraulic liquids for aircrafts, furniture's, aqueous film forming foam and textile industries [13]. Loos et. al.suggest that the electrostatic interaction plays an important role in the adsorption of perfluorocarbon (PFC) on minerals [14]. Wang et. al. studied the adsorption behavior of PFOA and reported that adsorption of PFOA decreases with increase in $\mathrm{pH}$ [15]. Xiaodong Gao et. al. studied molecular mechanism of PFOA adsorption at iron oxide surface [16].

We utilize this background knowledge to generate SAM of PFOA on mild steel surface by dip coating (DC-PFOA) and in a new approach; it is intended to generate an organic coat of PFOA by electrochemical method (EC-PFOA) at room temperature. Interestingly, the EC-PFOA showed remarkable enhancement in protection efficiency as compared to DC-PFOA. The probable reason for this enhancement in the corrosion protection efficiency of EC-PFOA has been discussed in this article.

\section{Experimental}

\section{Materials and sample preparation}

Mild steel (chemical composition-0.04\% C, $0.35 \% \mathrm{Mn}, 0.022 \% \mathrm{P}, 0.036 \% \mathrm{~S}$, and remaining iron) coupons of dimension $1 \mathrm{~cm}^{2}$ area were used as substrates for surface modification tests and for electrochemical studies. The PFOA (Sigma Aldrich) was used as received. The corrosive media $3.5 \% \mathrm{NaCl}$ was prepared in Milli-pore water (Elix 3 Milli-pore system).

Mild steel samples were abraded mechanically by using different grades of emery paper (220, $600,1000,2000,2500)$ and further sonicated with acetone and alcohol for $15 \mathrm{~min}$ to remove all debris followed by sonication in Milli-pore water for $20 \mathrm{~min}$. These samples were flushed with a stream of dry nitrogen gas and were kept in an ultraviolet cleaning chamber (Bioforce, Nanosciences, U.S.A.) for 30 min to burn all carbonaceous contaminants. Prior to modification process, unpolished samples (without cloth polishing) were washed with Milli-pore water followed by acetone and dried.

Two different techniques were used to generate PFOA coating on mild steel;

1. Dip coating - (DC-PFOA); the steel samples were dipped in aqueous solution of $2 \mathrm{mM}$ PFOA for 18 hours at room temperature. Then taken out, rinsed and washed with ethanol followed by $n$-hexane and dried.

2. Electrochemical coating - (EC-PFOA); the steel sample was dipped in PFOA solution of different concentrations (1, 2, 4 and $6 \mathrm{mM}$ aqueous solution) and anodized at $0.2 \mathrm{~V} v \mathrm{v}$. $\mathrm{Ag} / \mathrm{AgCl}$ using $\mathrm{CHI660C}$ electrochemical workstation (U.S.A.) for different time intervals 
(5, 15, 30 and $60 \mathrm{~min})$. The samples are taken out, rinsed and washed with ethanol followed by $n$-hexane and dried.

These samples were then subjected to corrosion studies in $3.5 \% \mathrm{NaCl}$ media.

Infrared Reflection Absorption Spectroscopy (IRRAS) analysis and contact angle measurement

IRRA spectra were obtained at room temperature using GX Spectrometer (Perkin Elmer, USA), equipped with a liquid nitrogen cooled mercury cadmium telluride (MCT) detector. Before starting the experiments the sample and detector chambers were purged with nitrogen gas to eliminate moisture content. All spectra were acquired at $4 \mathrm{~cm}^{-1}$ resolution and 1024 scans. A heating accessory (Harrick scientific corporation, New York, USA) was used for taking the spectra with an incident angle of $75^{\circ}$ from the surface normal. The spectral analysis was carried out by spectrum 3.02 version software (Perkin-Elmer, U.S.A.). Goniometer (OCA 30, Data Physics Instruments) was used to measure the water contact angle on steel at room temperature. $2 \mu \mathrm{L}$ of Millipore water was used to measure the angle by sessile drop method. Images of the air/liquid/solid (A/L/S) systems were captured and processed using the 32-bit SCA 20 software.

\section{Electrochemical studies}

Electrochemical studies were carried out using $\mathrm{CHI660C}$ electrochemical work station at room temperature. The steel samples of $1 \mathrm{~cm}^{2}$ area, platinum wire and $\mathrm{Ag} / \mathrm{AgCl}$ were used as working, counter and reference electrodes, respectively. The samples were immersed in $3.5 \% \mathrm{NaCl}$ for 400 seconds prior to electrochemical measurements in order to establish open circuit potential (OCP). The electrochemical impedance spectroscopy (EIS) measurements were carried out at OCP in the frequency range of $100 \mathrm{kHz}$ to $10 \mathrm{mHz}$ with $5 \mathrm{mV}$ sinusoidal excitation signal. EIS data were analyzed using ZSimpWin-3.21 software. The polarization measurements were carried out at a scan rate of $1 \mathrm{mV} \mathrm{s}^{-1}$.

\section{Surface morphology}

The surface morphology of the bare steel, coated steel and corroded steel was investigated using FESEM QUANTA 250 Scanning Electron Microscopy (SEM). The SEM images of corroded samples were taken after immersion of coated sample in $3.5 \% \mathrm{NaCl}$ for $6 \mathrm{~h}$ at room temperature. After the immersion, the samples were rinsed with distillated water and dried before imaging the samples by SEM. Secondary electron detector was used to obtain SEM micrographs. Elemental composition of the modified surface was performed by using Energy Dispersive X-ray Spectroscopy (EDS).

\section{Results and discussion}

\section{Characterization of coating}

IRRAS analysis

The IRRAS of adsorbed PFOA molecule on mild steel by dip coating method and electrochemical coating at room temperature are shown in Figure 1. Peak position and mode assignments are given in Table 1. The presence of $\mathrm{COO}^{\circ}, \mathrm{C}-\mathrm{C}$ and $\mathrm{C}-\mathrm{F}$ stretching modes in both the spectra confirms the adsorbed PFOA on steel surface [16]. The asymmetric stretch of deprotonated carboxylate head group $\left[v_{\text {as }}\left(\mathrm{COO}^{-}\right)\right]$is observed at $1683 \mathrm{~cm}^{-1}$ in both cases, whereas the $v_{\mathrm{s}}\left(\mathrm{COO}^{-}\right)$is shifted to slightly higher wavenumber $\left(1437 \mathrm{~cm}^{-1}\right)$ in EC-PFOA. For the EC-PFOA, there is a shift in adsorption band of $v_{\mathrm{ax}}\left(\mathrm{CF}_{2}\right)$ by $24 \mathrm{~cm}^{-1}$ compared to DC-PFOA in the direction of lower wavenumber. Despite 
the similarities between two spectra, the C-C stretching peaks are strong $\left(1104,1023 \mathrm{~cm}^{-1}\right)$ for ECPFOA which can be attributed to the compactness of the film.
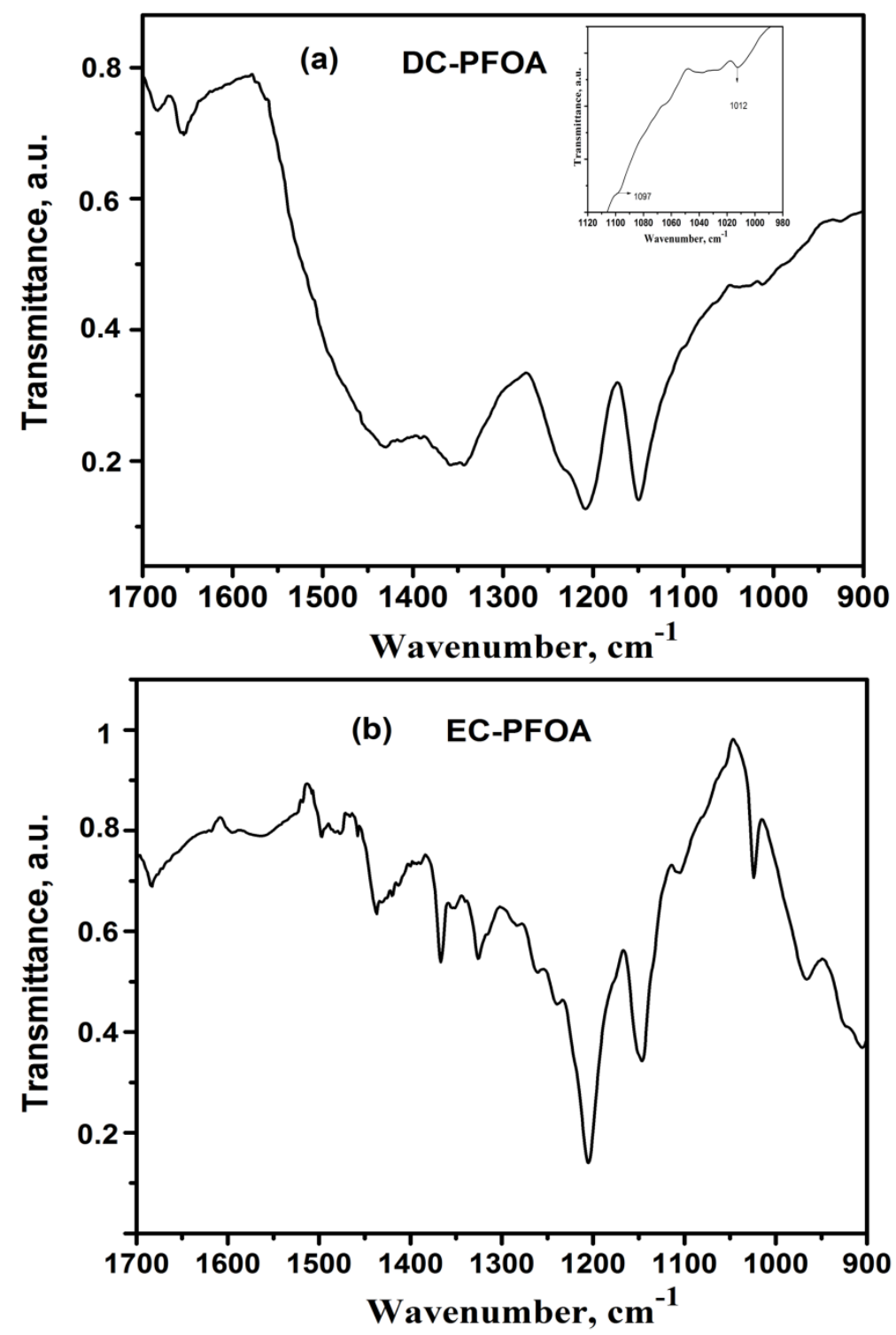

Figure 1. IRRA Spectra of PFOA on steel by (a) dip coating method (b) electrochemical coating method.

Table 1. IRRAS vibrational frequencies of PFOA deposited on mild steel substrate.

\begin{tabular}{lcc}
\hline & \multicolumn{2}{c}{ Wavenumber, $\mathbf{c m}^{\mathbf{- 1}}$} \\
\hline Mode assignment & DC-PFOA & EC-PFOA \\
\hline$v_{\text {ax }}\left(\mathrm{CF}_{2}\right)$ & 1342 & 1366 \\
$v_{\text {as }}\left(\mathrm{CF}_{2}\right)$ & 1233 & 1239 \\
$v_{\mathrm{s}}\left(\mathrm{CF}_{2}\right)$ & 1149 & 1146 \\
$v_{\text {as }}\left(\mathrm{CF}_{2}\right)+v_{\text {as }}\left(\mathrm{CF}_{3}\right)$ & 1208 & 1205 \\
$v_{\text {as }}\left(\mathrm{COO}^{-}\right)$ & 1683 & 1683 \\
$v_{\mathrm{s}}\left(\mathrm{COO}^{-}\right)$ & 1431 & 1437 \\
$v(\mathrm{C}-\mathrm{C})$ & 1097 & 1104 \\
$v(\mathrm{C}-\mathrm{C})$ & 1012 & 1023 \\
\hline
\end{tabular}


Contact angle measurement

Contact angle measurements are the simple and effective technique to quantify surface wetting properties. The hydrophilic, hydrophobic and super hydrophobic character of the surface can be evaluated with the help of contact angle developed between three phases; liquid, solid and gas [17]. Figure 2 shows the water contact angle $\left(\theta_{\text {water }}\right)$ on modified steel surface by (a) DC-PFOA (b) EC-PFOA at room temperature. The $\theta_{\text {water }}$ on DC-PFOA is $128^{\circ}$ and for EC-PFOA is $135^{\circ}$, which illustrates hydrophobic nature of the modified surface. The acid head group of PFOA anchors to the metal surface by electrostatic interaction and the non-polar perfluorocarbon chain protrudes towards the space, stabilized by lateral van der Waals force. The interaction between nonpolar tail group of PFOA and polar water molecule will be less, which leads higher contact angle $[12,18]$. The increase in the water contact angle for EC-PFOA by $7^{\circ}$ can be attributed to the compactness of the film which is a result of electrochemically driven oriented growth.

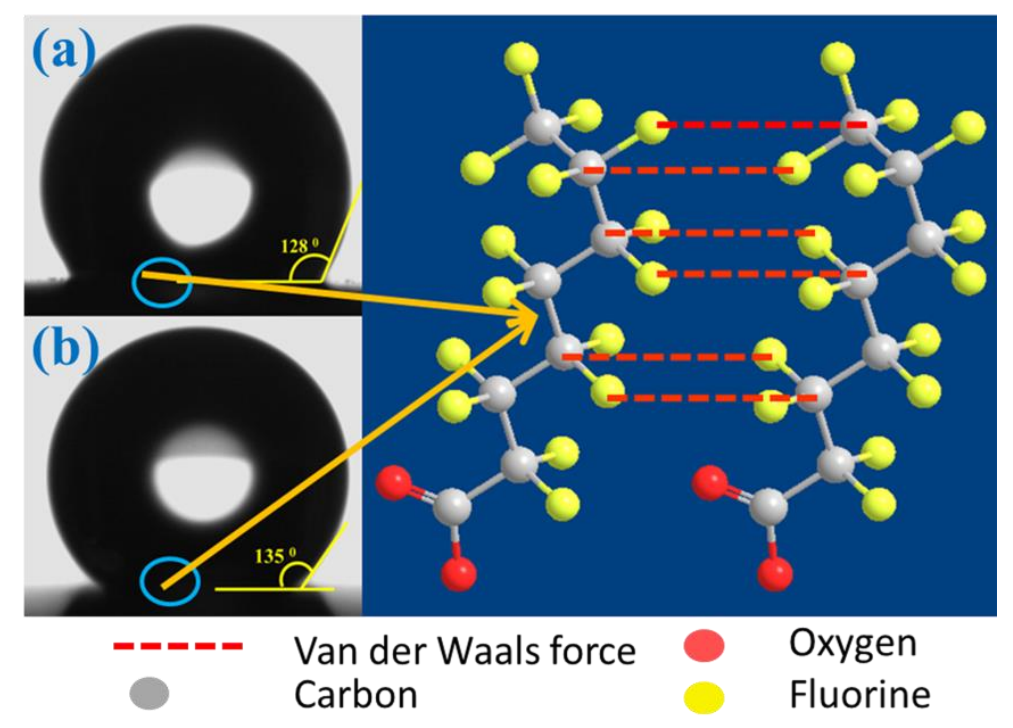

Figure 2. Water contact angle on (a) DC-PFOA (b) EC-PFOA at room temperature.

The $\theta_{\text {water }}$ for sodium oleate SAM on mild steel was $113^{\circ}[12]$, where the hydrocarbon chain has 18 carbon atoms. However, PFOA with only 8 carbon atoms exhibited higher contact angle. This may be due to the different structure of carbon chain. The hydrocarbon chain has zigzag $\mathrm{C}-\mathrm{C}-\mathrm{C}$ structure whereas perfluorocarbon has helical structure, which imparts rigidity to the molecule. This is because fluorine van der Waals radii $(0.147 \mathrm{~nm})$ which is more than hydrogen $(0.12 \mathrm{~nm})$ leads to $12-14^{\circ}$ twist of the F-C-F bond about the carbon backbone causes geometric and stearic hindrance [13].

\section{Electrochemical analysis}

\section{Electrochemical impedance analysis}

The EIS helps to quantitatively determine the coating properties over a wide frequency range. Moreover, it is a non-destructive and efficient tool to evaluate the corrosion behavior of organic coatings [19]. The impedance data were measured in $3.5 \% \mathrm{NaCl}$ solution and represented in the form of Nyquist plots. To analyze this acquired impedance data the electrical equivalent circuit (EEC) model given in Figure 3 has been used in all cases. It consists of solution resistance $\left(R_{\mathrm{s}}\right)$, coating resistance $\left(R_{\text {coat }}\right)$, charge transfer resistance $\left(R_{\mathrm{ct}}\right)$, coating capacitance $\left(Q_{\text {coat }}\right)$ and double 
layer capacitance $\left(Q_{\mathrm{dl}}\right)$. The constant phase element $(C P E)$ is replaced for the capacitor to fit the Nyquist loops more precisely. The impedance of the CPE is defined as

$$
Z j \omega=Q^{-1}(j \omega)^{-n}
$$

where, $j$ is the imaginary unit, $\omega$ is the angular frequency ( $\omega=2 \pi f, f$ is the frequency), $Q$ is the CPE constant and $n$ is the CPE exponent $(-1 \leq n \leq 1)$ where $Q$ and $n$ are frequency independent parameters. CPE defines an ideal capacitor for $n=1$, an ideal inductor for $n=-1$ and an ideal resistor for $n=0$ [20]. The decrease in the $n$ value signifies that the non-uniform distribution of current arises from surface roughness and surface defects [21].

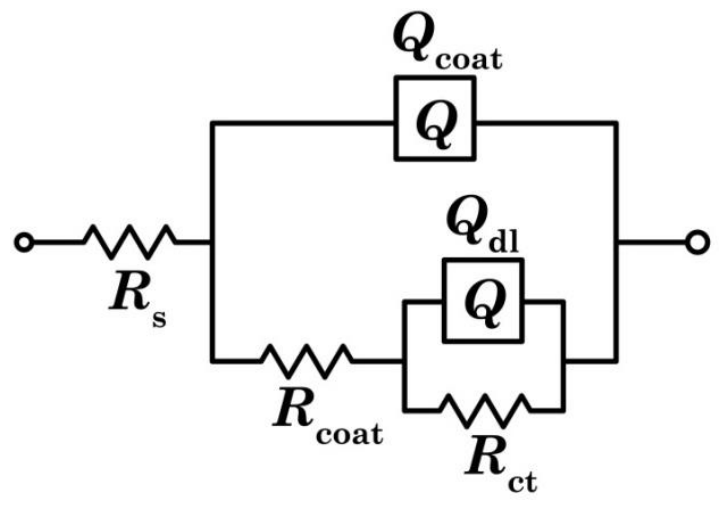

Figure 3. Electrical equivalent circuit used to fit the impedance measurements.

Figure 4 represents the Nyquist plots recorded for coated sample (DC-PFOA, EC-PFOA) immersed in $3.5 \% \mathrm{NaCl}$ solution at room temperature and the corrosion parameters calculated from the impedance measurement are tabulated in Table 2. The Figure 4a represents impedance measurement of bare and DC-PFOA immersed in $3.5 \% \mathrm{NaCl}$ solution at room temperature. Both (bare and DC-PFOA) the Nyquist loops are similar with respect to their shape but they vary slightly in their size and this observation reveals that the same course of action taking place with different magnitude at the metal/electrolyte interface. Each curve contains two semicircles one at higher and other at lower frequency end, indicating two relaxation processes is involved in the mechanism [22]. The electrochemical parameters tabulated in the Table 2 clears that the double layer capacitance decreases for DC-PFOA compare to bare sample. The slight increase in charge transfer resistance for DC-PFOA as compared to bare steel, infers there is no significant corrosion protection. The charge transfer resistance is proportional to exposed area by following equation [23]

$$
A_{\mathrm{e}}=R_{\mathrm{ct}}^{0} / R_{\mathrm{ct}}
$$

Where $A_{\mathrm{e}}$ is the exposed area, $R_{\mathrm{ct}}^{0}$ is the charge transfer resistance for bare steel and $R_{\mathrm{ct}}$ is the charge transfer resistance for DC-PFOA. The calculated exposed area from above equation is $71 \%$, this is due to the formation of weak or discontinuous SAM. The reason for insignificant protection from DC-PFOA can be explained as the following: the $\mathrm{pH}$ of aqueous PFOA solution is 4.5. The surface of steel has zero charge at $\mathrm{pH} 5.2$ to 6.7 , below this $\mathrm{pH}(<5.2)$ surface gets positive charge [24]. Though the surface has positive charge below isoelectric point, at $\mathrm{pH} 4.5$ it has feeble positive charge which doesn't facilitate effective adsorption. Hence, there is week adsorption between negatively charged acid head group of PFOA and the steel surface. Hence, the SAM thus generated is fragile and exhibits poor protection ability.

The Nyquist plots of EC-PFOA generated for $30 \mathrm{~min}$ (at various concentrations) immersed in $3.5 \% \mathrm{NaCl}$ solution at room temperature are shown in Figure 4b. Two distorted capacitive loops 
are observed in the Nyquist plot for all concentration curves. With increase in concentration from 1 to $2 \mathrm{mM}$, the double layer capacitance values decreased from 52.26 to $15.06 \mu \Omega^{-1} \mathrm{~cm}^{-2} \mathrm{~s}^{n}$ and charge transfer resistance values increases from 3533 to $12520 \Omega \mathrm{cm}^{2}$. During electrochemical coating $0.2 \mathrm{~V}$ potential was applied to the working electrode to increase the positive charge on the working electrode. The negatively charged head group of PFOA is thus directed to the steel surface due to electrostatic force of attraction. This forms a superior directional compact coating which gives excellent corrosion protection. The increase in double layer capacitance and decrease in the $\mathrm{n}_{2}$ value was observed for EC-PFOA generated in $4 \mathrm{mM}$ and other higher concentration PFOA solution. This indicates inhomogeneous and uneven coating.
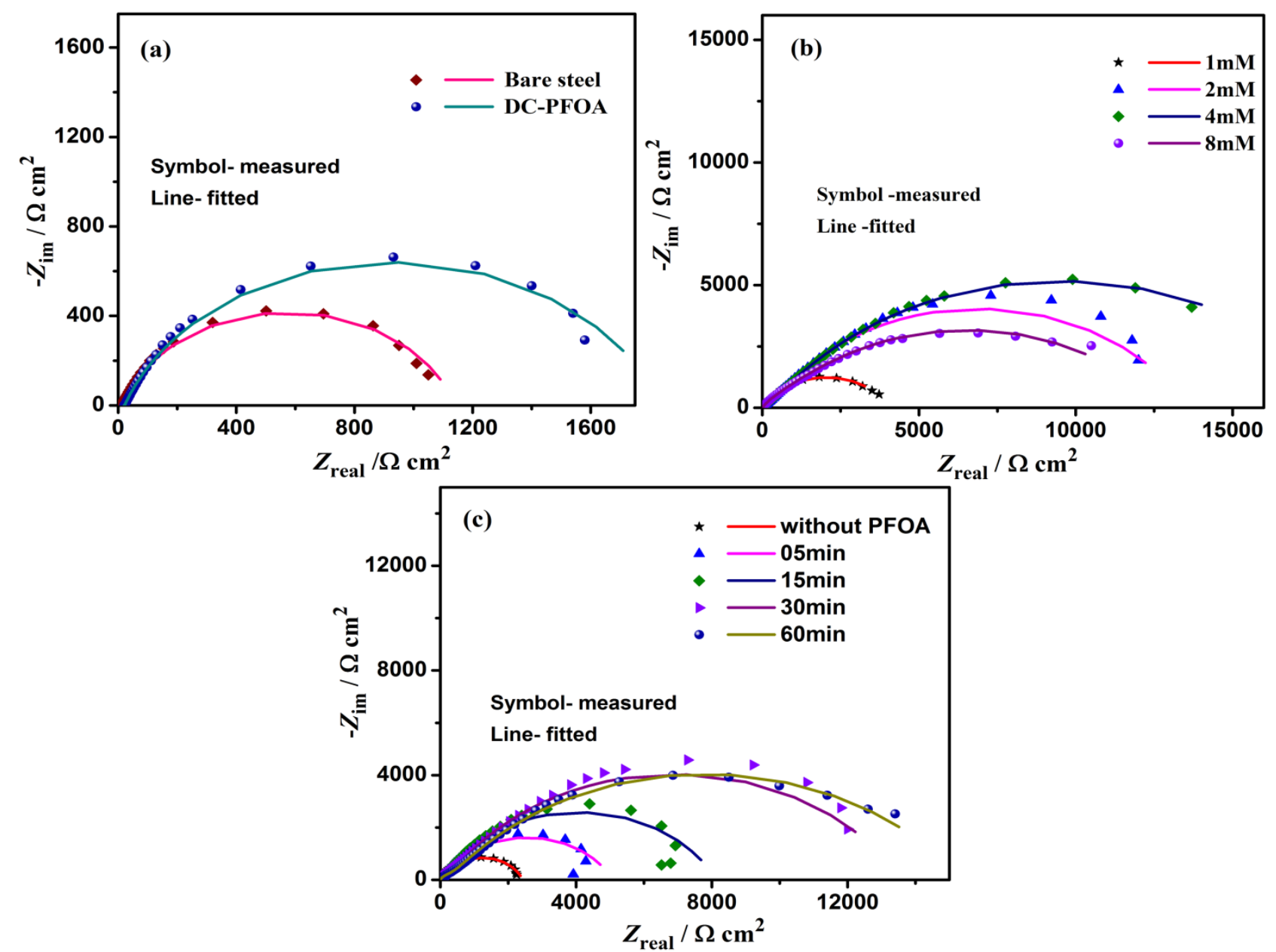

Figure 4. Nyquist plots of (a) DC-PFOA (b) EC-PFOA at various concentrations (for $30 \mathrm{~min}$ ) (c) EC-PFOA at different coating time (for $2 \mathrm{mM}$ PFOA solution), immersed in $3.5 \% \mathrm{NaCl}$ solution at room temperature.

The Nyquist diagram of EC-PFOA generated in $2 \mathrm{mM}$ solution (at different coating time) immersed in $3.5 \% \mathrm{NaCl}$ solution at room temperature is represented in Figure 4c. The curves seem to be similar with respect to their shape indicating the similarity in mechanistic electrochemical property of the films. However, it should be noted that they differ substantially in their size. The area enclosed by the loop indicates extent of corrosion resistance. With increasing time, the Nyquist loops of EC-PFOA samples enclose larger area with higher charge transfer resistance. The charge transfer resistance is related to protection efficiency $(P E)$ by the following equation [25]:

$P E / \%=\left[\left(R_{\mathrm{ct}}-R_{\mathrm{ct}}^{0}\right) / R_{\mathrm{ct}}\right] \times 100$ 
Table 2. Corrosion data obtained from EIS technique.

\begin{tabular}{|c|c|c|c|c|c|c|c|}
\hline Sample & $Q_{\text {coat }} / \mu \Omega^{-1} \mathrm{~cm}^{-2} \mathrm{~S}^{\mathrm{n}}$ & $\mathrm{n}_{1}$ & $R_{\text {coat }} / \Omega \mathrm{cm}^{2}$ & $Q_{\mathrm{dl}} / \mu \Omega^{-1} \mathrm{~cm}^{-2} S^{n}$ & $\mathrm{n}_{2}$ & $R_{\mathrm{ct}} / \Omega \mathrm{cm}^{2}$ & Error, \% \\
\hline Bare steel & 249 & 0.8 & 7 & 376 & 0.8 & 01124 & 2.7 \\
\hline DC-PFOA & 14 & 0.4 & 22 & 4 & 0.8 & 01575 & 2.7 \\
\hline \multicolumn{8}{|c|}{ EC-PFOA generated in various concentrations (at $30 \mathrm{~min}$ ). } \\
\hline $1 \mathrm{mM}$ & 145 & 0.6 & 566 & 52 & 0.8 & 03533 & 1.8 \\
\hline $2 \mathrm{mM}$ & 15 & 0.7 & 1051 & 15 & 0.7 & 12520 & 4.2 \\
\hline $4 \mathrm{mM}$ & 12 & 0.7 & 919 & 21 & 0.6 & 13094 & 1.2 \\
\hline $6 \mathrm{mM}$ & 23 & 0.7 & 2481 & 36 & 0.6 & 10280 & 1.6 \\
\hline \multicolumn{8}{|c|}{ EC-PFOA generated in different coating time (at $2 \mathrm{mM}$ PFOA solution) } \\
\hline Without SAM & 93 & 0.8 & 29 & 72 & 0.8 & 02393 & 2.4 \\
\hline $5 \mathrm{~min}$ & 41 & 0.7 & 56 & 52 & 0.8 & 05015 & 4.3 \\
\hline $15 \mathrm{~min}$ & 36 & 0.7 & 712 & 12 & 0.8 & 07418 & 4.4 \\
\hline $30 \mathrm{~min}$ & 15 & 0.7 & 1051 & 15 & 0.7 & 12520 & 4.2 \\
\hline $60 \mathrm{~min}$ & 6 & 0.8 & 506 & 48 & 0.6 & 14860 & 2.2 \\
\hline
\end{tabular}

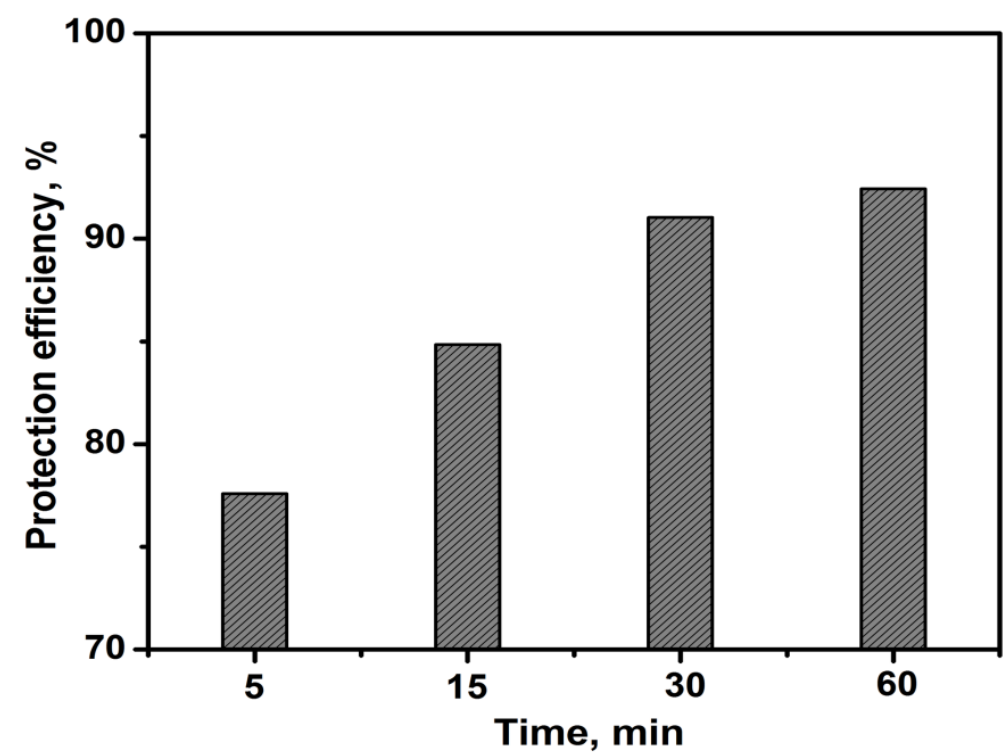

Figure 5. Corrosion protection efficiency of EC-PFOA at different coating time in $3.5 \% \mathrm{NaCl}$ solution at room temperature.

where, $R_{\text {ct }}^{0}$ and $R_{\text {ct }}$ represent the charge-transfer resistance of bare and coated sample respectively. The protection efficiency of EC-PFOA for different coating time is shown in Figure 5. The PE increases with increase in coating time. However, beyond $30 \mathrm{~min}$ there is no significant increase in PE. The double layer capacitance of EC-PFOA decreases up to $30 \mathrm{~min}$ and then increases (60 min). The $\mathrm{n}_{2}$ value also decreases for 60 min represents surface inhomogeneity. The charge transfer resistance for uncoated sample (bare steel kept in water without PFOA for $30 \mathrm{~min}$ ) in $3.5 \% \mathrm{NaCl}$ solution is $2393 \Omega \mathrm{cm}^{2}$, but for coated sample (30 min immersion in PFOA solution) is $12520 \Omega \mathrm{cm}^{2}$. This clearly indicates that the EC-PFOA blocks the active site and thus reduces the anodic reaction more efficiently than DC-PFOA due to strong electrostatic interaction between steel and PFOA molecule.

Potentiodynamic polarization analysis

The Tafel plots of coated sample (DC-PFOA, EC-PFOA) immersed in $3.5 \% \mathrm{NaCl}$ solution at room temperature are displayed in Figure 6 . The values of the corrosion potential $\left(E_{\text {corr }}\right)$, corrosion current density $\left(I_{\text {corr }}\right)$ and corrosion rate were tabulated in Table 3 . The Figure $6 a$ shows the 
polarization behavior of bare steel and DC-PFOA immersed in $3.5 \% \mathrm{NaCl}$ solution at room temperature. The $I_{\text {corr }}$ values for bare steel and DC-PFOA are $6.091 \mu \mathrm{A} \mathrm{cm}$ and $5.558 \mu \mathrm{A} \mathrm{cm}$, respectively. There is no significant decrease in either corrosion current density value or corrosion rate values. This shows that the DC-PFOA has poor protection efficiency.
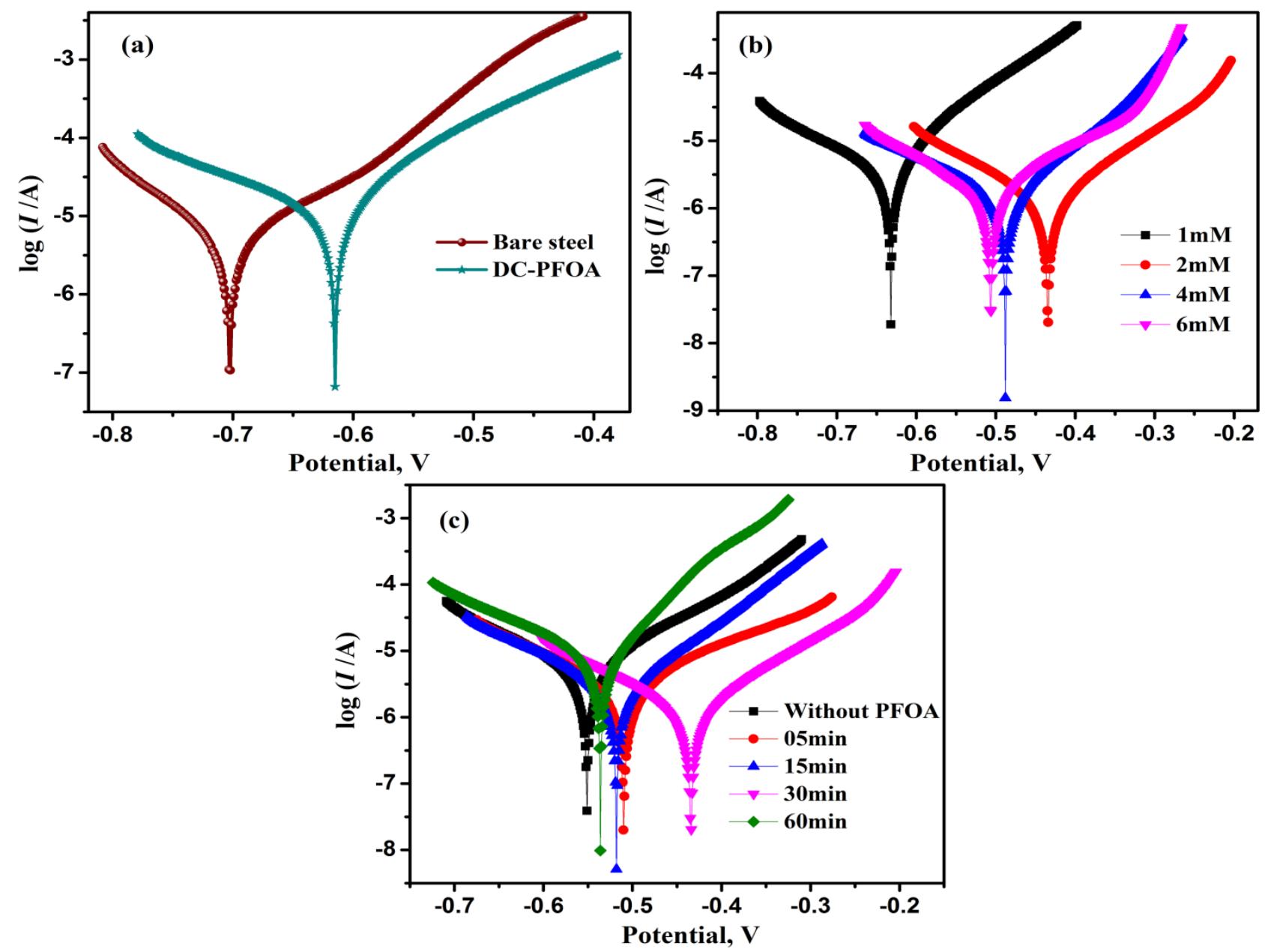

Figure 6. Tafel plots of (a) DC-PFOA (b) EC-PFOA at various concentrations (for $30 \mathrm{~min}$ ) (c) C-PFOA at different coating time (for $2 \mathrm{mM} P F O A$ solution), immersed in $3.5 \% \mathrm{NaCl}$ solution at room temperature.

Table 3. Corrosion data obtained from Tafel measurement.

\begin{tabular}{cccc}
\hline Sample & $\boldsymbol{E}_{\text {corr }} / \mathbf{V}$ & $\boldsymbol{I}_{\text {corr }} / \boldsymbol{\mu A} \mathbf{~ c m}^{-2}$ & Corrosion rate, mils/yr \\
\hline Bare steel & -0.702 & 6.09 & 5.57 \\
DC-PFOA & -0.615 & 5.56 & 5.08 \\
EC-PFOA generated in various concentrations (at 30 min). \\
$1 \mathrm{mM}$ & -0.632 & 4.77 & 4.36 \\
$2 \mathrm{mM}$ & -0.434 & 1.50 & 1.37 \\
$4 \mathrm{mM}$ & -0.488 & 1.85 & 1.69 \\
$6 \mathrm{mM}$ & -0.506 & 2.19 & 2.00 \\
EC-PFOA generated in different coating time (at 2 $\mathbf{m M}$ PFOA solution) \\
Without PFOA & -0.551 & 5.50 & 5.03 \\
$5 \mathrm{~min}$ & -0.510 & 3.32 & 3.04 \\
$15 \mathrm{~min}$ & -0.518 & 3.02 & 2.76 \\
$30 \mathrm{~min}$ & -0.434 & 1.50 & 1.37 \\
$60 \mathrm{~min}$ & -0.536 & 5.46 & 4.99 \\
\hline
\end{tabular}


The Tafel plots of EC-PFOA generated for $30 \mathrm{~min}$ (in different PFOA concentrations) immersed in $3.5 \% \mathrm{NaCl}$ solution at room temperature are presented in Figure $6 \mathrm{~b}$. The $I_{\text {corr }}$ and corrosion rate value of EC-PFOA decreases to a large extent from 1 to $2 \mathrm{mM}$, then again increases with concentration. Meanwhile, $E_{\text {corr }}$ values for coating in $2 \mathrm{mM}$ PFOA also shifted towards more positive direction compared to coatings at other concentrations. This indicates that the surface coverage was more effective in $2 \mathrm{mM}$ concentration, thus decreases the dissolution rate of steel remarkably.

The Tafel plots of EC-PFOA generated in 2mM PFOA solution (at different coating time) immersed in $3.5 \% \mathrm{NaCl}$ solution at room temperature are shown in Figure $6 \mathrm{c}$. The $I_{\text {corr }}$ and corrosion rate values decreases with increase in coating time and the lowest value observed for 30 min coating time. Moreover, the $E_{\text {corr }}$ value also shifted towards positive side prominently for 30 min coating time. This implies that the EC-PFOA generated for $30 \mathrm{~min}$ has significant effect on inhibiting the anodic dissolution.

\section{Surface morphology}

Scanning electron microscopic images were taken to establish the formation of protective layer on the mild steel surface. The SEM images of bare steel, DC-PFOA and EC-PFOA before and after immersion in $3.5 \% \mathrm{NaCl}$ for $6 \mathrm{~h}$ at room temperature are depicted in Figure 7.

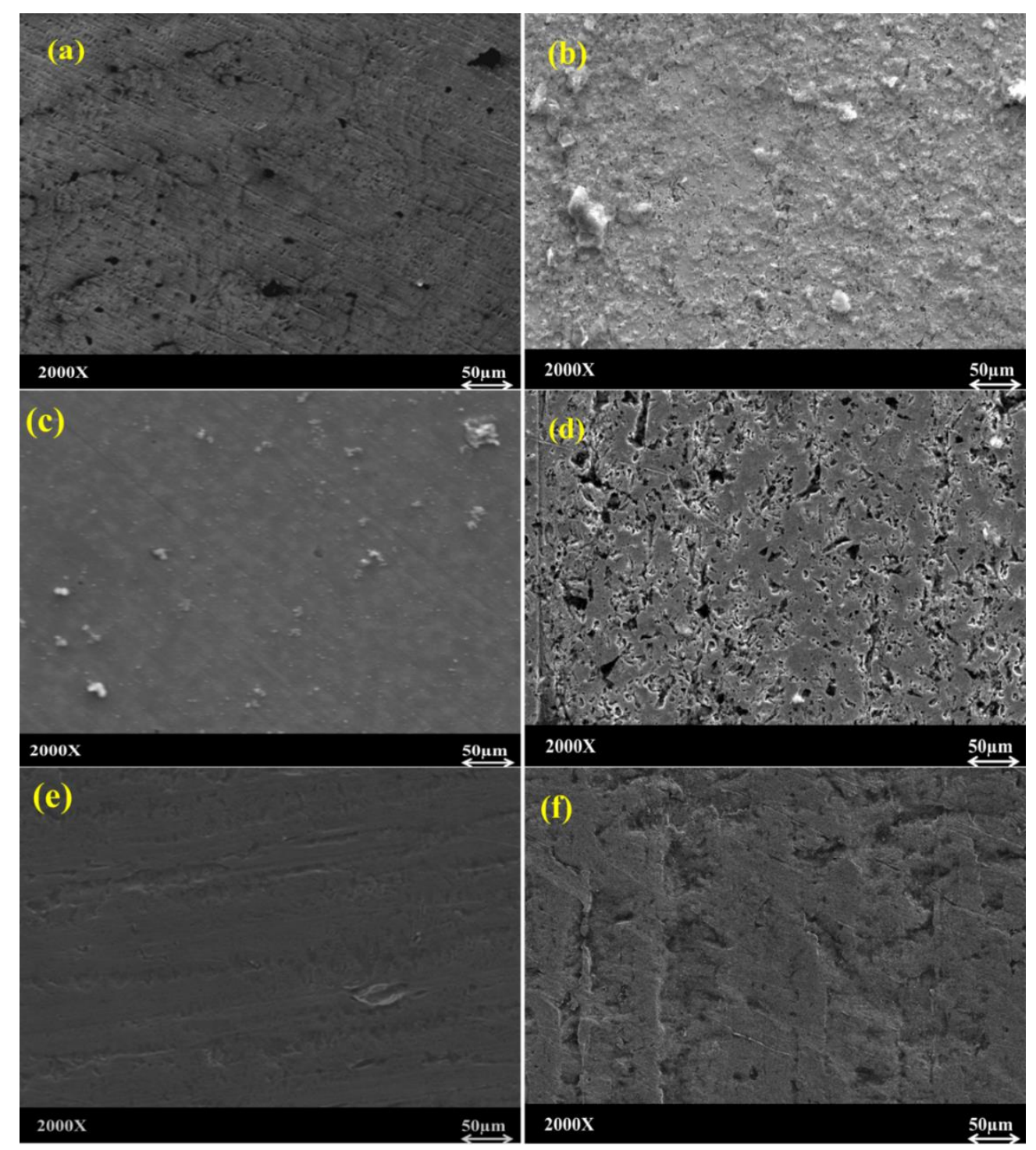

Figure 7. SEM micrographs of (a) unpolished bare steel (b) bare steel after corrosion (c) DCPFOA (d) DC-PFOA after corrosion (e) EC-PFOA (f) EC-PFOA after corrosion. 
The significant corrosion debris can be seen on the surface of bare steel after immersion in corrosive media (Figure 7a and 7b). PFOA molecules were dip coated giving smooth surface compared to bare steel surface which can be observed from Figure 7c. Figure 7d shows the surface morphology of DC-PFOA after corrosion. The pits formed on the surface imply the poor protection from DC-PFOA. Interestingly the electrochemically coated surface turns out to be smoother one morphologically (Figure 7e). However the EC-PFOA after immersion in corrosive medium (Figure 7f) has only small notches with almost smooth surface indicate the remarkable protection. This advocates the significant corrosion protection ability of electrochemically generated PFOA.

The protective film formed on the mild steel surface was analyzed using EDS as shown in Figure 8. Figure 8a represents EDS spectra of DC-PFOA. EDS spectra confirms the presence of iron (base metal, 79.76 wt. \%), carbon (11.55 wt. \%), oxygen (3.06 wt. \%) and Fluorine (5.63 wt. \%). Figure $8 \mathrm{~b}$ represents EDS spectra of EC-PFOA. The spectra confirms the presence of iron (80.26 wt. \%), carbon (11.09 wt. \%), oxygen (3.12 wt. \%) and Fluorine (5.53 wt. \%). The observed elemental composition from EDS confirms that PFOA molecule precludes the corrosion of surface by blocking the flaws.
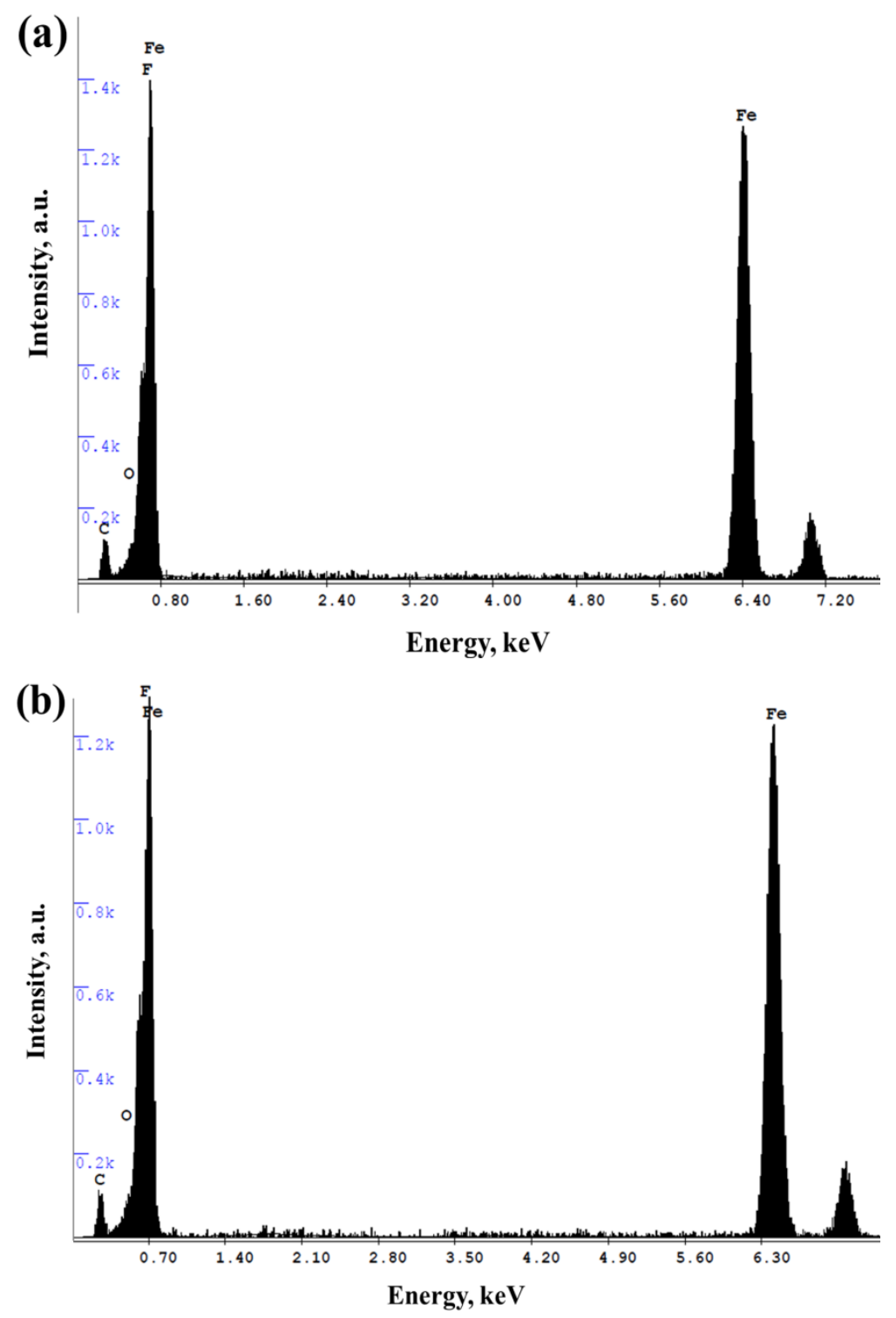

Figure 8. EDS of (a) DC-PFOA (b) EC-PFOA 


\section{Conclusions}

It is clearly evident from the above discussion that the electrochemical method of surface modification of mild steel is an effective way to protect the steel surface from the attack of corrosive agents. The electrochemical method provides a directional orientation to the organic film growth. The organic coat (EC-PFOA) thus generated is more compact and exhibits high hydrophobicity, which is evident from the well-defined IRRAS peaks and higher water contact angle $\left(\theta_{\text {water }}\right.$ for DC-PFOA is $128^{\circ} \theta_{\text {water }}$ for EC-PFOA is $\left.135^{\circ}\right)$. The electrochemical studies provide the ground support for these observations. The $R_{c t}$ for DC-PFOA coated in $2 \mathrm{mM}$ solution for $18 \mathrm{~h}$ is $1575 \Omega \mathrm{cm}^{2}$ but for 30 min coated EC-PFOA in $2 \mathrm{mM}$ solution for is $12520 \Omega \mathrm{cm}^{2}$. The EC-PFOA exhibited remarkable protection efficiency $(91 \%)$ as compared to DC-PFOA (28\%) due to strong electrostatic interaction.

Acknowledgements: The authors are grateful to Department of Chemistry, Kuvempu University, Karnataka, India, for providing lab facilities. The authors also thank to Prof. Satish V. Kailas, Mechanical Engineering Department, Indian Institute of Science, Bangalore, Karnataka, India, for providing instrumental facilities. We acknowledge the help given by Mr. H. S. Shamasundar, Dr. M. K. Punith Kumar, Dr. S. Ranganatha, and Mrs. M. K. Pavithra in conducting different experiments.

\section{References}

[1] C. E. Chuka, B. O. Odio, J. L. Chukwuneke, J. E. Sinebe, International Journal of Scientific \& Technology Research 3 (2014) 306-310.

[2] J. Gulicovski, J. Bajat, V. Stanković, B. Jokić, V. Panić, S. Milonjić, J. Electrochem. Sci. Eng. 3 (2013) 151-156.

[3] M. A. Chidiebere, E. E. Oguzie, L. Liu, Y. Li, F. Wanga, J. Ind. Eng. Chem. 26 (2015) 182-192.

[4] R. Lopes-Sesenes, J.G. Gonzalez-Rodriguez, G. F. Dominguez-Patiño, A. Martinez-Villafañe, J. Electrochem. Sci. Eng. 2 (2012) 77-90.

[5] O. P. Khatri, S. K. Biswas, Surf. Sci. 600 (2006) 4399-4404.

[6] S. Ramachandran, B. L. Tsai, M. Blanco, H. Chen, Y. Tang, W. A. Goddard III, Langmuir 12 (1996) 6419-6428.

[7] F. Rosei, M. Schunack, Y. Naitoh, P. Jiang, A. Gourdon, E. Laegsgaard, I. Stensgaard, C. Joachim, F. Besenbacher, Prog. Surf. Sci. 71 (2003) 95-146.

[8] F. Schreiber, Prog. Surf. Sci. 65 (2000) 151-256.

[9] H. Luo, Y.C. Guan, K.N. Han, Corros. Sci. 54 (1998) 619-627.

[10] K. Nozawa, H. Nishihara, K. Aramaki, Corros. Sci. 39 (1997) 1625-1639.

[11] P. E. Hintze, L. M. Calle, Electrochim. Acta. 51 (2006) 1761-1766.

[12] H. N. Shubha, T. V. Venkatesha, V. Kanagalasara, M. K. Pavithra, M. K. Punithkumar, ACS Appl. Mater. Interfaces. 5 (2013) 10738-10744.

[13] D.M. Lemal, J. Org. Chem. 69 (2004) 1-11.

[14] R. Loos, G. Locoro, T. Huber, J. Wollgast, E.H. Christoph, A.D. Jager, B.M. Gawlik, G. Hanke, G. Umlauf, J.M. Zaldívar, Chemosphere 71 (2008) 306-313.

[15] F. Wang, K. Shih, water research 45 (2011) 2925-2930.

[16] X. Gao, J. Chorover, Environ. Chem. 9 (2012) 148-157.

[17] T. T. Chau, W.J. Bruckard, P. T. L. Koh, A. V. Nguyen, Adv. Colloid Interface Sci. 150 (2009) 106-115.

[18] M. J. Oh, S. Y. Lee, K. H. Paik, J. Ind. Eng. Chem. 17 (2011) 149-153.

[19] T. Monetta, F. Bellucei, L. Nicodemo, L. Nicolais, Prog.Org. Coat. 21 (1993) 353-369.

[20] A. K. Mishra, R. Balasubramaniam, S. Tiwari, Anti-Corros.Methods Mater. 54 (2007) 37-46.

[21] M. K. Punithkumar, T. V. Venkatesha, M. K. Pavithra, N. A. Shetty, Phys. Scr. 84 (2011) 1-10. 
[22] S. Ranganatha, T. V. Venkatesha, K. Vathsala, Ind. Eng. Chem. Res. 51 (2012) 7932-7940.

[23] J. M. McIntyre, H.Q. Pham, Prog. Org. Coat. 27 (1996) 201-207.

[24] K. R. Henke, Arsenic Environmental chemistry, Health threts and waste treatment, Wiley, U.K. 2009, 48.

[25] Z. Zhang, S. Chen, Y. Li , S. Li , L. Wang, Corros. Sci. 51 (2009) 291-300.

(C) 2015 by the authors; licensee IAPC, Zagreb, Croatia. This article is an open-access article distributed under the terms and conditions of the Creative Commons Attribution license (http://creativecommons.org/licenses/by/4.0/) 